\title{
Antisperm antibodies disrupt plasma membrane integrity and inhibit tyrosine phosphorylation in human spermatozoa
}

\author{
Dwi A. Pujianto, ${ }_{1}^{1}$ Hajizah, ${ }^{2}$ Indra G. Mansur, ${ }^{1}$ Amarudin ${ }^{1}$ \\ ${ }^{1}$ Department of Biology, Faculty of Medicine, Universitas Indonesia, Jakarta, Indonesia \\ ${ }^{2}$ Master Program for Biomedical Sciences, Faculty of Medicine, Universitas Indonesia, Jakarta, Indonesia
}

\section{ABSTRACT}

Background: The etiology of unexplained infertility has not been fully understood. This study aimed to determine the effect of antisperm antibody (ASA) from infertile women on viability, motility, plasma membrane integrity, and status of tyrosine phosphorylation in the human spermatozoa.

Methods: An experimental in vitro study was conducted at the Department of Biology, Faculty of Medicine, Universitas Indonesia from February to November 2014. Spermatozoa from normal fertile donors was incubated with serum containing ASA from infertile women at several dilutions $(1 / 1000,1 / 100,1 / 10$, and without dilution) for 1 and 2 hours. The plasma membrane integrity was assessed with hypoosmotic swelling (HOS) test, whereas the status of tyrosine phosphorylation was analyzed using Western immunoblotting and immunocytochemistry.

Results: After 1 hour incubation time, ASA caused a decrease in sperm viability, motility, plasma membrane integrity, and inhibit sperm tyrosine phosphorylation. ASA caused a decrease in viability, motility, sperm plasma membrane integrity, and tyrosine phosphorylation of sperm after 1 hour incubation time.

Conclusion: ASA from infertile women reduced the sperm viability, motility, plasma membrane integrity, and capacitation in dose and time dependent manner.

Keywords: antisperm antibody, plasma membrane integrity, tyrosine phosphorylation pISSN: 0853-1773• eISSN: 2252-8083・https://doi.org/10.13181/mji.v27i1.1429 • Med J Indones. 2018;27:3-11

- Received 17 Apr 2017 • Accepted 14 Dec 2017 
Spermatozoa are cells that possess little cytoplasm and organelles. To be able to fertilize oocytes, sperm should have normal shapes and move progressively. After being ejaculated, mammalian sperm do not have full capability to fertilize the oocyte. They need to undergo a series of physiological and biochemical changes that render capability to penetrate the oocyte. These changes are collectively called capacitation. Capacitation causes spermatozoa to get hyperactive motility, interact with zona pellucida (ZP), and undergo acrosome reaction to fuse with plasma membrane of the oocytes. ${ }^{1}$ Antisperm antibodies (ASA) present in blood serum, seminal plasma, and female reproductive tract have long been suspected interfering sperm function. ${ }^{2}$ A recent proteomic analysis showed that certain sperm proteins are capable of inducing antisperm antibodies ${ }^{3}$ that interfere sperm function including inhibition of motility, viability, ${ }^{4}$ and acrosome reaction, ${ }^{2,5}$ block the interaction of sperm with oocytes (fertilization) at a specific stage, ${ }^{6}$ and cause low sperm binding to the oocyte. ${ }^{7}$

The mechanism of ASA inhibition on sperm capacitation process has not been elucidated at the molecular level. Previous studies have shown that during capacitation there is an increase in tyrosine phosphorylation. ${ }^{8}$ Phosphorylation of proteins on tyrosine residues is the main indicator of ongoing process of capacitation, and the process requires signal transduction through high integrity of sperm plasma membrane. ${ }^{9}$ The effect of ASA on sperm tyrosine phosphorylation and plasma membrane integrity associated with sperm capacitation is largely unknown. Whether the presence of ASA inhibits capacitation which ultimately blocks or prevents the fertilization process remains to be elucidated for better diagnosis and therapy of male infertility. This study aimed to analize the effect of antisperm antibodies from infertile patients towards sperm motility, viability, membrane integrity, and status of the tyrosine phosphorylation.

\section{METHODS}

\section{Sperm samples and antisperm antibodies}

Serum containing antisperm antibodies used in this study were obtained from Sam Marie Wijaya Fertility Hospital, Sayidah Mother and
Child Hospital, and Andrology Laboratory at the Department of Biology, Faculty of Medicine, Universitas Indonesia. Serums were collected from women with unexplained infertility. There were 22 serums from infertile women. All serums had been tested positive for ASA by mixed agglutination reaction (MAR) test. Six serums with high titer (based on MAR test) were subsequently used to incubate sperm cells throughout this study. Serum and human sperm from volunteer were obtained with informed consent. Donor sperm were in normal range after being examined by standard sperm analysis according to the World Health Organization (WHO). All the sample collecting methods have been approved by Ethic Committee of the Faculty of Medicine, Universitas Indonesia No 78/112.F1/ETIK/2013.

\section{Preparation of spermatozoa}

Sperm were obtained from fertile donors by means of masturbation after abstinence for at least 2 days. Sperm were collected in the sterile containers made of glass and left at room temperature for 20 minutes to liquefy. The examinations were performed to confirm normal parameters such as ejaculation volume, $\mathrm{pH}$ and color of semen, concentration, viability, motility, plasma membrane integrity, and agglutination. Sperm were washed by centrifugation at 2,000 rpm for 15 minutes in 50\% Percol to separate sperm cells from seminal plasma. Supernatant was removed, and the pellet containing cells was washed with $3 \mathrm{~mL}$ of biggers whitten whittingham (BWW) medium. Sperm were collected with another centrifugation at $2,000 \mathrm{rpm}$ for 10 minutes. Pellet from the second centrifugation which contained pure spermatozoa was then resuspended in $1 \mathrm{ml}$ of BWW, and the concentration was determined using a Neubauer counting chamber. For further experiments, spermatozoa were diluted at final concentration of $10 \times 10^{6}$ spermatozoa $/ \mathrm{mL}$ in BWW medium.

\section{Sperm incubation with ASA}

After being washed, sperm samples were divided into 4 groups according to the concentration or dilution of the serum. Sperm were incubated in $500 \mu \mathrm{l}$ BWW medium in the presence of serum containing antisperm antibodies at zero dilution (as a control), 1/1000, $1 / 100$ and $1 / 10$ dilution ratios. Incubation was performed in $1.5 \mathrm{~mL}$ microcentrifuge tube and 
put in an incubator at $37^{\circ} \mathrm{C}$ for 1 hour and 2 hours. After incubation, sperm cells were divided for analyses on viability, motility, hypo-osmotic swelling (HOS) test, Western immunoblotting, and immunocytochemistry.

\section{Examination of spermatozoa viability}

Ten microliters of semen were mixed with $10 \mu \mathrm{L}$ of eosin-Y solution and was put onto the microscope slide. Then it was covered with a deckglass. Counting of life and death cells was performed under magnification of 400 times. The percentage of life sperm was determined from a total of 100 sperm cells. The life sperm was white because the plasma membrane was still intact, while the dead sperm was red because the plasma membrane was no longer able to withstand the eosin solution.

\section{Examination of sperm motility}

Ten microliters of the samples were applied on microslides of Neubauer counting chamber and were observed under a light microscope with a magnification of 400 times. The percentage of motile sperm was calculated from a total of 100 cells.

\section{Sperm membrane integrity assay}

After incubation, sperm plasma membrane integrity was assessed using HOS test method. HOS test was performed by mixing 0.1 $\mathrm{ml}$ of sperm sample with $1.0 \mathrm{ml}$ of $150 \mathrm{mmol} /$ $\mathrm{kg}$ of hypo-osmotic solution, which was made by mixing $7.35 \mathrm{~g}$ of sodium citrate and $13.5 \mathrm{~g}$ of fructose in $1000 \mathrm{ml}$ of distilled water. The mixture was incubated for 60 minutes at $37^{\circ} \mathrm{C}$ in a waterbath. Then $0.2 \mathrm{ml}$ of the mixture was placed on a slide and mounted with a coverslip and observed immediately at 400x magnification using a light microscope. The percentage of sperm plasma membrane integrity was measured by counting spermatozoa with intact plasma membrane (indicated by curly or bent sperm tail) and disrupted plasma membrane (indicated by straight tail) divided by total number of sperm counted.

\section{Examination of sperm tyrosine phosphorylation}

Western blot analysis was performed to examine the effect of ASA on sperm tyrosine phosphorylation which indicated the status of sperm capacitation. After incubation with
ASA, sperm proteins were extracted using SDS extraction buffer (2\% SDS, $10 \%$ sucrose, 0.1875 $\mathrm{M}$ Tris $\mathrm{pH}$ 6.8). Samples were centrifuged at $13,000 \mathrm{rpm}$ for 5 minutes. Supernatant was removed, while the pellet was solubilized in $35 \mu \mathrm{l}$ SDS extraction buffer. Samples were then boiled for 5 minutes and centrifuged at $13,000 \mathrm{rpm}$ for 10 minutes. The supernatant containing sperm proteins was transferred to a new tube. Ten micrograms of sperm proteins were separated by $10 \%$ SDS PAGE at a voltage of 100 volts for 1.5 hours, followed by a transfer to polyvinylidene difluoride (PVDF) membranes (GE Healthcare, UK) at a voltage of 100 volts for 1.5 hours. The membrane was then blocked with $3 \%$ bovine serum albumin (BSA) for one hour at room temperature. Membrane was incubated in 1\% BSA containing Monoclonal Anti-Phosphotyrosine antibody (Sigma, St. Louis, USA) with a concentration of $1: 2,000$ overnight at $4^{\circ} \mathrm{C}$. The next day, the membrane was washed with $1 \mathrm{x}$ tris-buffered saline-tween 20 (TBST) for $3 \times 5$ min and then incubated with goat antimouse IgG-HRP conjugated (Santa Cruz, USA) at a concentration of 1:5,000 for two hours at a room temperature. The membrane was then washed again with 1xTBST then visualized with ECL Plus Western blot detection system (GE Healthcare, UK).

\section{Localization of sperm tyrosine phosphorylation}

Examination of tyrosine phosphorylation on sperm cell was carried out using immunocytochemistry. After incubation, samples were put into fixative solution $(2 \%$ paraformaldehyde (PFA)) for 30 minutes, washed with phosphate-buffered saline (PBS) and stored at $4^{\circ} \mathrm{C}$ for one night. The next day, the suspension of spermatozoa was air-dried on polylysine-coated slides by pipetting $50 \mu \mathrm{l}$ suspension of each sample on the slide and incubated in a moist chamber for 2 hours. Spermatozoa that attached to the slide were then incubated for 10 minutes in 0.1 Triton X-100 for cell solubilization. The slides were blocked for 30 minutes using a solution of $3 \%$ bovine serum albumin (BSA). The slides were then incubated with Monoclonal Anti-Phosphotyrosine which recognized phosphorylated tyrosine residues for overnight at $4^{\circ} \mathrm{C}$. The slides were then washed 3 times with TBST and followed by incubation in secondary antibody which was Goat Anti-Mouse 
Immunoglobulin Goat Anti-Mouse G-Fluorescein isothiocyanate (IgG-FITC) conjugated (Santa Cruz, USA). After 3 times of washing with TBST, the cells were mounted with mowiol and covered with a cover glass and observed under a fluorescent microscope.

\section{Statistical analysis}

Statistical package for the social sciences (SPSS) 20.0 was used for statistical analysis. Parametric test of one-way analysis of varian (ANOVA) was used for the measurement of viability, motility, plasma membrane integrity of spermatozoa, and tyrosine phosphorylation. $\mathrm{P} \leq 0.05$ was considered statistically significant difference.

\section{RESULTS}

\section{Examination of donor semen samples}

The analysis of donor's semen comprising volume, $\mathrm{pH}$, color, concentration, viability, motility, and membrane integrity is presented in Table 1. These results show the normal characteristics according to the World Health Organization (WHO) standard of semen analysis.

\section{ASA decreased sperm viability and motility}

Sperm viability after incubation with ASA was examined by using Eosin-Y staining method. The result was observed using a light microscope. The image of live spermatozoa is white because the plasma membrane is still intact, while the dead spermatozoa are red because the plasma membrane was disrupted so that it could not prevent eosin solution from entering the cell as shown in Figure 1.

Table 1. Average parameters of donor semen used in the study (Numbers in parenthesis indicate range of normal values)

\begin{tabular}{lc}
\hline Description & Results \\
\hline Volume (ml) & $3.5(2.5-4.5)$ \\
pH & $7.4(7.3-7.5)$ \\
Colour & White grey \\
Consentration (million/ml) & $84(39-198)$ \\
Viability (\%) & $72(55-95)$ \\
Motility (\%) & $67(50-83)$ \\
Membrane integrity (\%) & $75(70-83)$ \\
Agglutination (\%) & $4(0-7)$ \\
\hline
\end{tabular}

The percentage of sperm viability was obtained by counting the number of live sperm (white) from a total of 100 cells that were counted (Figure 2A). The result showed that sperm viability decreased after 1 hour incubation with ASA at 1:10 dilution. To verify whether there were significant differences in sperm viability in each treatment group, we performed a statistical analysis. The results of one way ANOVA showed the value of $p=0.690$ ( $p>0.05$ ) indicating there was no statistically

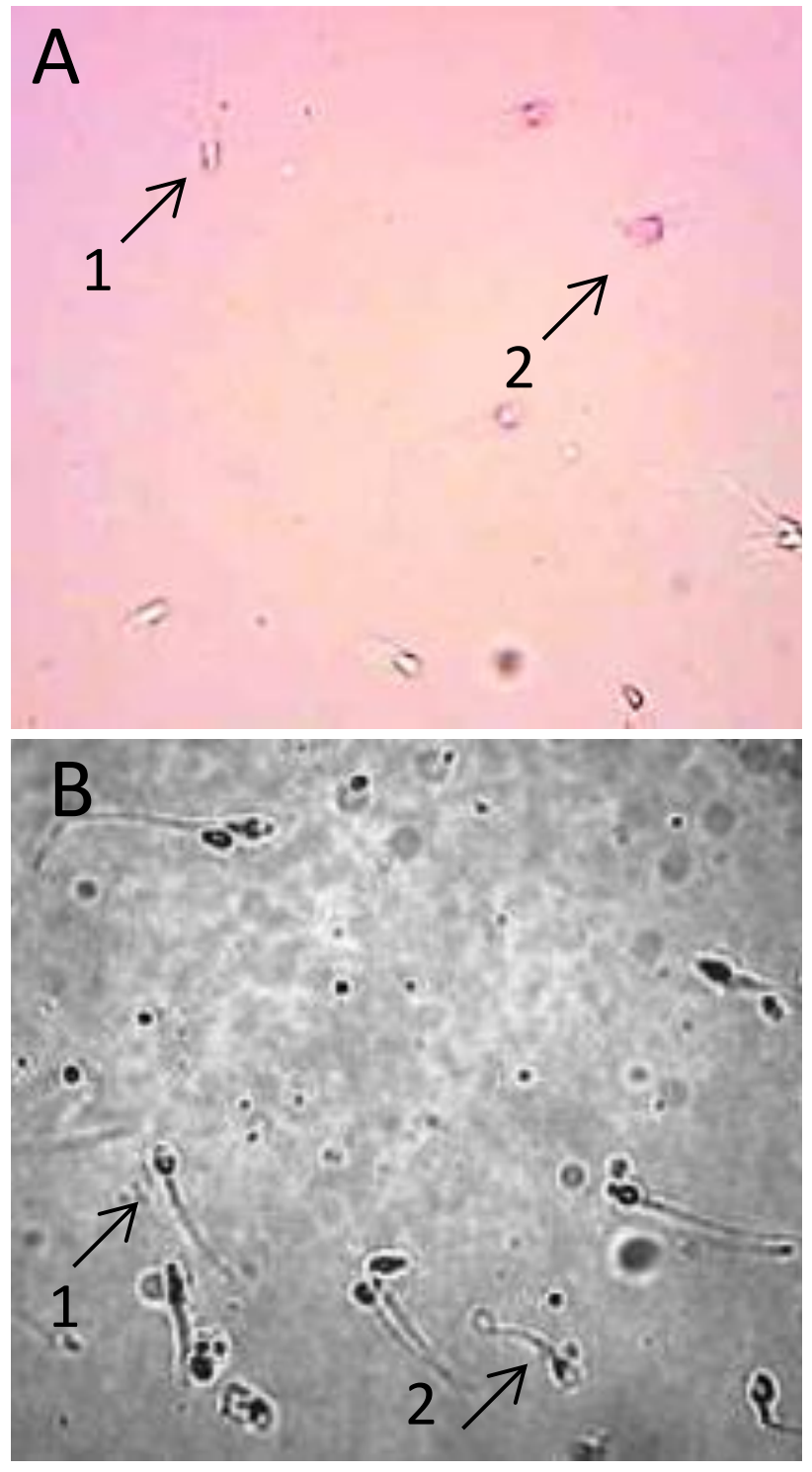

Figure 1. A) Image of viability assay showing of live sperm (A.1) and the dead sperm (A.2) after incubation with ASA. Sperm were stained with Eosin-Y; B) Sperm plasma membrane integrity in hypo-osmotic swelling test. Sperm with leaked plasma membrane were observed with straight tail (B.1), whereas sperm with intact plasma membrane show curly tail (B.2). Sperm cells were observed under a light microscope at 400x magnification 
significant difference in the sperm viability in each treatment group.

In contrast to sperm viability, sperm motility decreased after 1 hour incubation with ASA at dilution of $1 / 10$. Incubation for 2 hours decreased sperm motility, but statistically it was not significant $(\mathrm{p}=0.759)$ as seen in Figure 2B.

\section{ASA decreased sperm membrane integrity}

Assessment of sperm plasma membrane integrity after being incubated with ASA was performed using hypoosmotic swelling (HOS) test. Sperm with intact membrane were indicated by curly tail, whereas leaked or disrupted sperm membrane was indicated by stright tail (Figure 1B). The result showed that plasma membrane integrity of spermatozoa diminished along with the increased serum concentration in the incubation for 1 hour (Figure 2C).

\section{ASA inhibited sperm tyrosine phosphorylation}

To investigate the effect of ASA on human sperm capacitation, total sperm protein was extracted from sperm cells, separated into polyacrylamide gel, transferred onto a PVDF membrane, and detected with an antibody recognizing total tyrosine phosphorylation. The result showed that ASA, at dilution of $1 / 10$, inhibited tyrosine phosphorylation at 1 hourincubation (Figure 3A). The effect was even more obvious after 2 hours incubation, in which tyrosine phosphorylation was reduced even at dilution of 1/1000 (Figure 3B).

To subsequently assess the distribution of tyrosine phosphorylation directly on sperm cells, immunocytochemistry was performed on sperm after being incubated with several dilutions of ASA. The result showed that tyrosine phosphorylation was detected in the mid-piece and principal piece of spermatozoa. Illustration of sperm was obtained from observations using a fluorescent microscope at $400 x$ magnification (Figure 4).

The percentage of sperm cells labelled with tyrosine phosphorylation was counted to examine whether ASA would affect number of cell undergoing
A
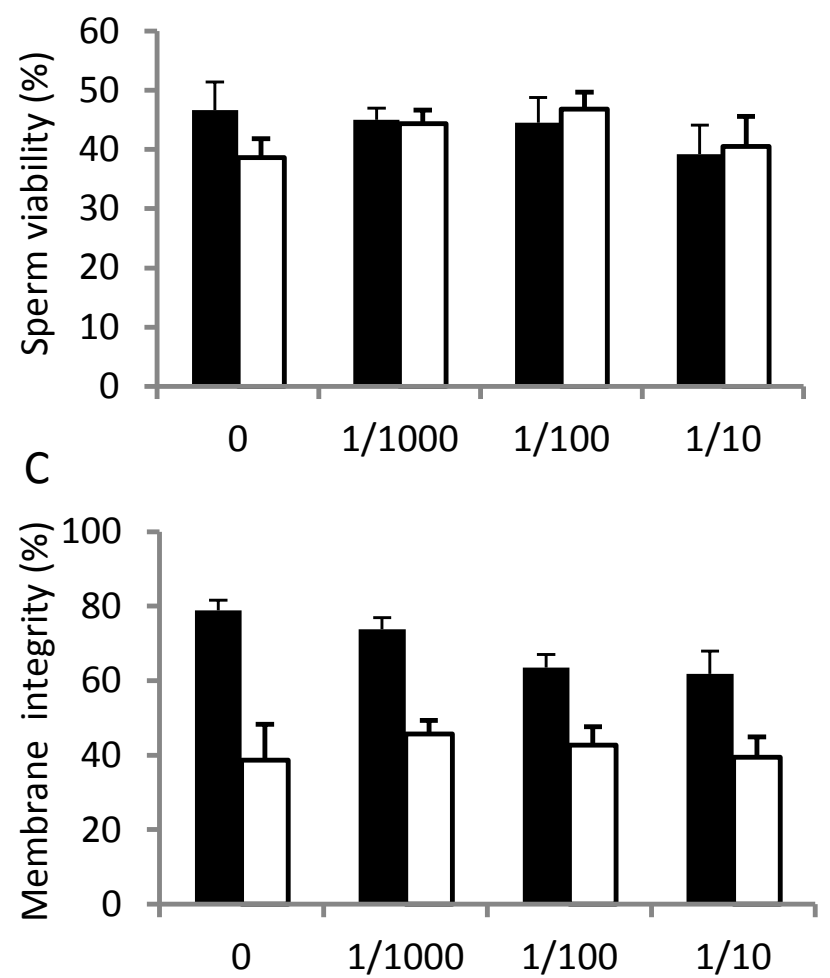

B

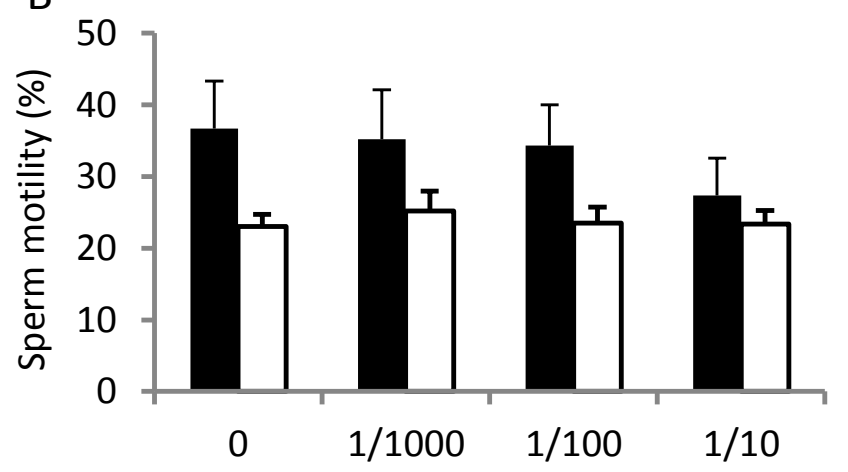

D

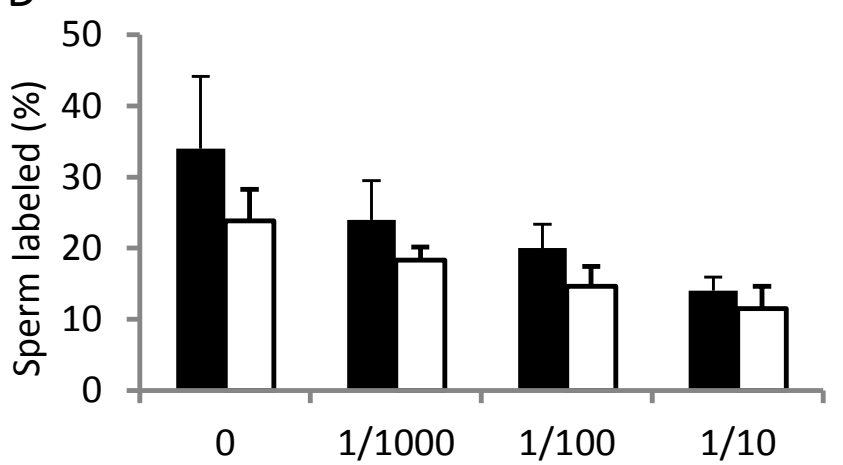

Figure 2. Percentage of sperm viability (A), motility (B), membrane integrity (C) and sperm labeled by anti phophotyrosine antibody (D) in the control group ( $0=$ without treatment) and after treatment with serum containing antisperm antibody at $1 / 1000$, $1 / 100$ and $1 / 10$ dilutions for 1 hour (black bars) and 2 hours (white bars) incubation at $37^{\circ} \mathrm{C}$. Error bars represent scanning electron microscopy (SEM) $(n=6)$. 


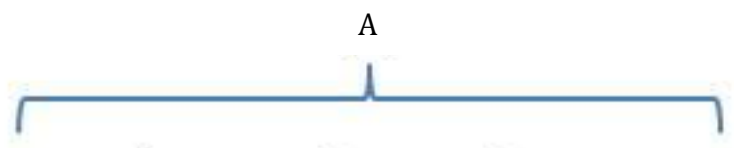

1

2

3

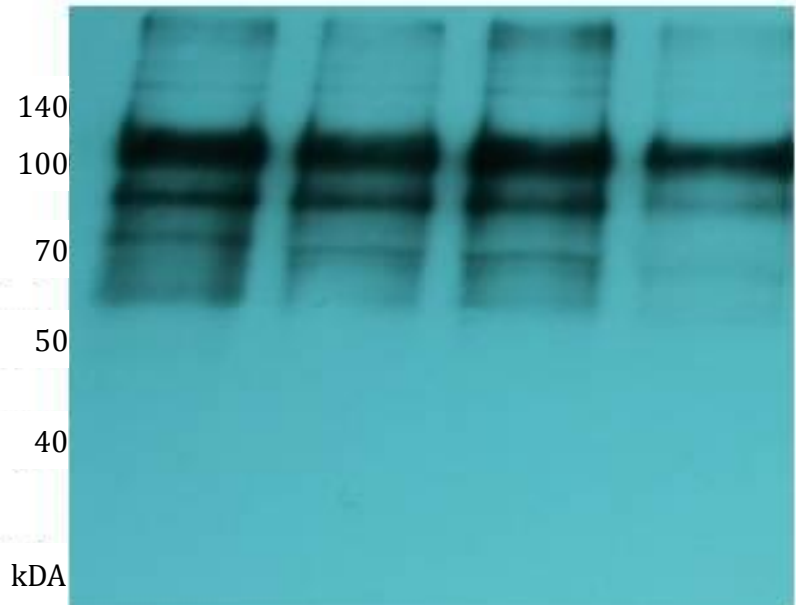

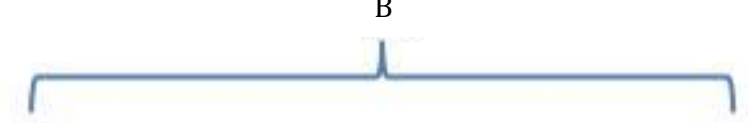

1

2

3

4

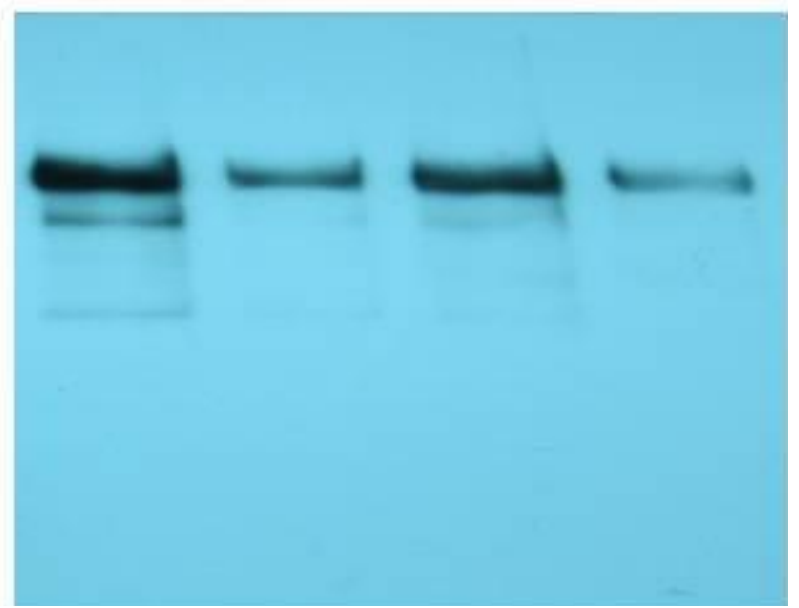

Figure 3. Western immunoblot analysis of tyrosine phosphorylation. Sperm were incubated without ASA as a control (1); and with ASA at 1/1000 (2), 1/100 (3) and 1/10 (4) dilution for $1 \mathrm{~h} \mathrm{(A)}$ and 2 hours (B). Cells were then solubilised in SDS extraction buffer, separated by $10 \%$ SDS-PAGE and transferred onto PVDF membrane. Proteins on membrane were then detected using phosphodetect antibody recognizing total tyrosine phosphorylation
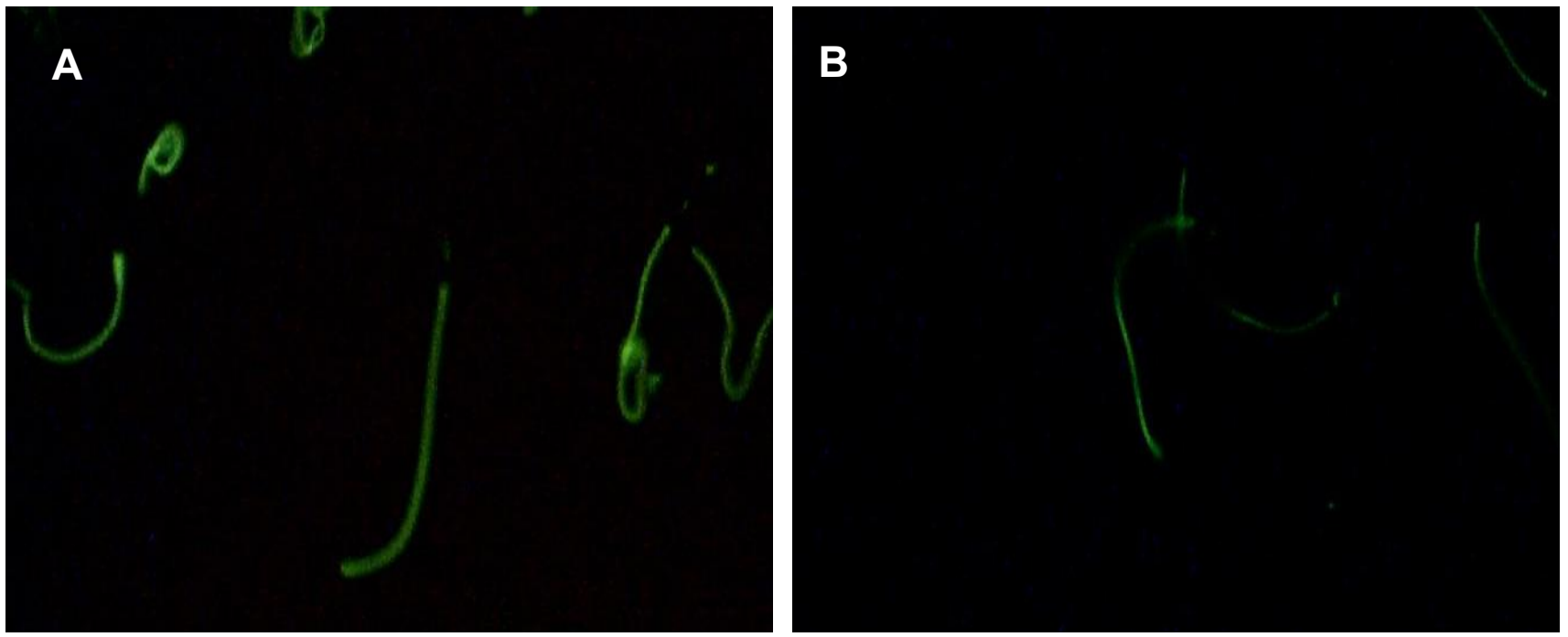

Figure 4. Immunocytochemistry analyses of sperm tyrosine phosphorylation in the control group (without ASA, panel A) and after incubation with ASA at dilution of 1/1000 (panel B). Tyrosine phosphorylation was observed mainly at the sperm tail and the number of sperm labelled was clearly reduced after incubation with ASA. (Tyrosine phosphorylation was observed using a fluoresent microscope at 1000x magnification)

capacitation. The result showed that ASA, at all dilutions and time used, reduced the number of cell labelled with tyrosine phosphorylation as presented in Figure 2D, confirming inhibition of ASA towards sperm capacitation.

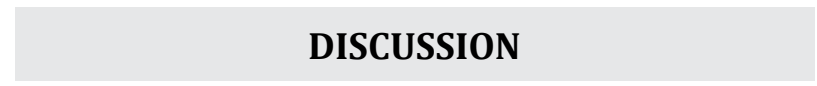

This study aimed to understand the mechanism of how antisperm antibodies disrupt 
sperm function especially on capacitation as indicated by tyrosine phosphorylation. Capacitation is an orchestrated process involving transduction of signal from extracellular environment into intracellular in which cell membrane integrity is an important requirement. This study was conducted by incubating normal sperm from donor with serum containing antisperm antibodies from infertile women.

This study showed that viability of spermatozoa slightly decreased along with increased concentration of ASA in 1-hour incubation. This phenomena was not observed in the 2-hour group. This indicates that the ASA could interrupt the sperm viability as reported by Bozhedomov and Veron. 2,10 The decline of sperm viability in group with higher ASA concentrations was thought to be related to the disruption of the integrity of the plasma membrane. Rossato et $\mathrm{l}^{11}$ reported that the HOS test scores in ASA-positive sperm was lower than in the negative group and positively correlated with the percentage of motility, viability, and morphology of spermatozoa. This is possible because the ASA bond to the sperm surface may interfere sperm membrane fluidity that leads to disruption of sperm capacitation. ${ }^{7}$ Another possibility is the interactions between sperm antigens and ASA that causes a variety of effects, among others are precipitation, agglutination, and inducing cell damage by stimulating immune responses such as activation of the classical complement pathway, especially the classical pathway which led to cell lysis. ${ }^{12}$

Sperm cells have a progressive and hyperactive motility. Sperm progressive motility is obtained during the process of maturation in the epididymis, characterized by a strong flagellar movement and relatively symmetrical producing rapid forward movement. Hyperactive motility was obtained in the female reproductive tract following the process of capacitation. Key factors involved in the initiation of progressive and hyperactive motility are already known, such as calcium ions, bicarbonate ions, and cyclic adenosine monophosphate (cAMP). ${ }^{13}$ Several studies reported the relationship between ASA with the motility of spermotozoa. Nakagawa et $\mathrm{al}^{14}$ reported that sperm immobilizing antibodies reduced sperm motility by suppressing internalization of alkyl ester lysophospholipid that led to disruption of sperm membrane fluidity, thus it also blocked capacitation. On the other hand, Rossato et al ${ }^{11}$ hypothesized that ASA binding to spermatozoa blocked water channel and modified the plasma membrane leading to disruption of the calcium influx necessary for sperm hyperactivation. This indicates that the ASA could interrupt sperm motility by compromising plasma membrane integrity. The integrity and fluidity of plasma membrane are essential in controlling the level of intracellular $\mathrm{Ca}$ involved in the initiation of sperm motility. This is supported by Lodi et $\mathrm{al}^{15}$ where they reported a positive correlation between HOS test score and sperm motility.

The incubation of spermatozoa with positive ASA serum showed a decline on plasma membrane integrity along with increased concentration of ASA for 1 hour incubation. Previous studies have shown that plasma membrane integrity correlated positively with viability and motility of spermatozoa. ${ }^{11,15}$ In this study, ASA binding may block channel of certain molecules causing plasma membrane modification. This modification altered plasma membrane permeability. Current data clearly shows a significant difference in plasma membrane integrity between the control and the treatment group with one hour incubation, but not in the two-hour incubation time. Thus, we hypothesize that antisperm antibodies disrupt sperm plasma membrane integrity in dose and time dependent manner.

This study also showed a decrease in the intensity of tyrosine phosphorylation as ASA concentration increased in both 1-hour and 2 -hour incubation. Phosphorylation of proteins is initiated by changes in membrane fluidity due to the efflux of cholesterol from the plasma membrane. ${ }^{16}$ Benhoff et al $^{17}$ reported that the cholesterol content in the control group was lower than the group exposed to ASA. This indicates that ASA affects membrane cholesterol subsequently prevents changes in membrane fluidity. This is also supported by study in the bovine sperm in which ASA inhibits changes in the plasma membrane fluidity associated with capacitation and binding of spermatozoa to the zona pellucida. ${ }^{7}$ In this study, the decrease in sperm tyrosine phosphorylation which is an 
indicator of capacitation, demonstrates that ASA disturbs the capacitation process by disrupting membrane integrity.

In the current study, phosphorylated proteins have molecular weight between 60 $\mathrm{kDa}-140 \mathrm{kDa}$. The major bands are the ones with molecules weight of $110 \mathrm{kDa}$ and $93 \mathrm{kDa}$, which are a-kinase anchoring protein 3 (AKAP3) and a-kinase anchoring protein 4 (AKAP4) proteins. These proteins are components of sperm fibrous sheath surrounding outer dense fiber that form axoneme. ${ }^{18}$ AKAP3 and AKAP4 are phosphorylated during capacitation, and it is important in regulating sperm motility. The presence of antisperm antibodies may inhibit activation of AKAP3 and AKAP4 as indicated by reduced band intensity in the western blot and also in the immunocytochemistry analyses (Figure 3-4). Both methods used antibody recognizing phosphorylated proteins at tyrosine residue. Our data is in accordance with study by Ferrer et $\mathrm{al}^{7}$ in which spermbound immunoglobulin A (IgA) inhibits sperm membrane potential change that occurs during capacitation in the bovine sperm detected by merocyanine 540 staining. This also occurs in human where ASA levels are proportionate to the sperm hyperactivation event causing capacitation disorder. ${ }^{2}$

The limitation of this study is that no specific class of antisperm antibodies is known. Determination of immunoglobulin class in the antisperm antibodies would give a better knowledge on inhibition mechanism. It is also interesting to observe phosphorylated proteins in the 2-D gel electrophoresis, since in this study we could not identify proteins that have very close molecular weight.

As a conclusion, this study is the first to show that serum containing ASA from infertile women disrupts sperm viability, motility, and plasma membrane integrity as well as inhibits sperm capacitation indicated by reduction of tyrosine phosphorylation. Further studies are needed to confirm which antibody subclass is the most harmful towards spermatozoa.

\section{Conflict of interest:}

The authors affirm no conflict of interest in this study.

\section{Acknowledgments}

We thank DRPM Universitas Indonesia for providing part of the research grant. We also thank Rukmana for providing sperm donors and performing sperm analysis.

\section{REFERENCES}

1. Gervasi MG, Visconti PE. Molecular changes and signaling events occurring in spermatozoa during epididymal maturation. Andrology. 2017;5:204-18.

2. Bozhedomov VA, Nikolaeva MA, Ushakova IV, Lipatova NA, Bozhedomova GE, Sukhikh GT. Functional deficit of sperm and fertility impairment in men with antisperm antibodies. J Reprod Immunol. 2015;112:95-101.

3. Nowicka-Bauer K, Kamieniczna M, Cibulka J, UlcovaGallova Z, Kurpisz M. Proteomic identification of sperm antigens using serum samples from individuals with and without antisperm antibodies. Andrologia. 2016;48:693-701.

4. Marey MA, Yousef MS, Kowsar R, Hambruch N, Shimizu $\mathrm{T}$, Pfarrer $\mathrm{C}$, et al. Local immune system in oviduct physiology and pathophysiology: attack or tolerance? Domest Anim Endocrinol. 2016;56:S204-11.

5. Vazquez-Levin MH, Marin-Briggiler CI, Veaute C. Antisperm antibodies: invaluable tools toward the identification of sperm proteins involved in fertilization. Am J Reprod Immunol. 2014;72:206-18.

6. Taneichi A, Shibahara H, Takahashi K, Sasaki S, Kikuchi $\mathrm{K}$, Sato I, et al. Effects of sera from infertile women with sperm immobilizing antibodies on fertilization and embryo development in vitro in mice. Am J Reprod Immunol. 2003;50:146-51.

7. Ferrer MS, Klabnik-Bradford J, Anderson DE, Bullington AC, Palomates RA, Miller LMJ, et al. Sperm-bound antisperm antibodies prevent capacitation of bovine spermatozoa. Theriogenology. 2017;89:58-67.

8. Naresh S,AtrejaSK.Theprotein tyrosine phosphorylation during in vitro capacitation and cryopreservation of mammalian spermatozoa. Cryobiology. 2015;70:211-6.

9. Visconti PE, Krapf D, de la Vega-Beltran JL, Acevedo JJ, Darszon A. Ion channels, phosphorylation and mammalian sperm capacitation. Asian J Androl. 2011;13:395-405.

10. Veron GL, Molina RI, Tissera AD, Estofan GM, MarinBriggiler CI, Vazquez-Levin MH. Incidence of sperm surface autoantibodies and relationship with routine semen parameters and sperm kinematics. Am J Reprod Immunol. 2016;76:59-69.

11. Rossato M, Galeazzi C, Ferigo M, Foresta C. Antisperm antibodies modify plasma membrane functional integrity and inhibit osmosensitive calcium influx in human sperm. Hum Reprod. 2004;19:1816-20.

12. Koyama K, Ito K, Hasegawa A. Role of male reproductive tract CD52 (mrt-CD52) in reproduction. Soc Reprod Fertil Suppl. 2007;63:103-10.

13. Hereng TH, Elgstoen KB, Eide L, Rosendal KR, Skalhegg BS. Serum albumin and HCO3- regulate separate pools of ATP in human spermatozoa. Hum Reprod. 2014;29:918-30. 
14. Nakagawa K, Yamano S, Kamada M, Maegawa M, Tokumura A, Irahara M, et al. Sperm-immobilizing antibodies suppress an increase in the plasma membrane fluidity of human spermatozoa. Fertil Steril. 2004;82(Suppl 3):1054-8.

15. Lodi L, Zubair M, Qureshi I, Ahmad I, Jamil H. Correlation between hypo-osmotic swelling test and various conventional semen evaluation parameters in fresh Nili Ravi buffalo and Sahiwal cow bull semen. Pak Vet J. 2008;28:186-8.

16. Boerke A, Tsai PS, Garcia-Gil N, Brewis IA, Gadella BM. Capacitation-dependent reorganization of microdomains in the apical sperm head plasma membrane: functional relationship with zona binding and the zona-induced acrosome reaction. Theriogenology. 2008;70:1188-96.

17. Benoff S, Cooper GW, Hurley I, Mandel FS, Rosenfeld DL. Antisperm antibody binding to human sperm inhibits capacitation induced changes in the levels of plasma membrane sterols. Am J Reprod Immunol. 1993;30:113-30.

18. Carr DW, Newell AE. The role of A-kinase anchoring proteins (AKaps) in regulating sperm function. Soc Reprod Fertil Suppl. 2007;63:135-41. 